R. DE GASPARO, O, HALGAS, D, HARANGOZO, M. KAISER*, E. F, PAI*, R, L. KRAUTHSIEGEL, F. DIEDERICH* (ETH ZURICH, SWITZERLAND)

Targeting a Large Active Site: Structure-Based Design of Nanomolar Inhibitors of Trypanosoma brucei Trypanothione Reductase Chem. Eur.J. 2019, 25, 11416-11421.

\title{
Nanomolar Inhibitor of Trypanosoma brucei Trypanothione Reductase
}
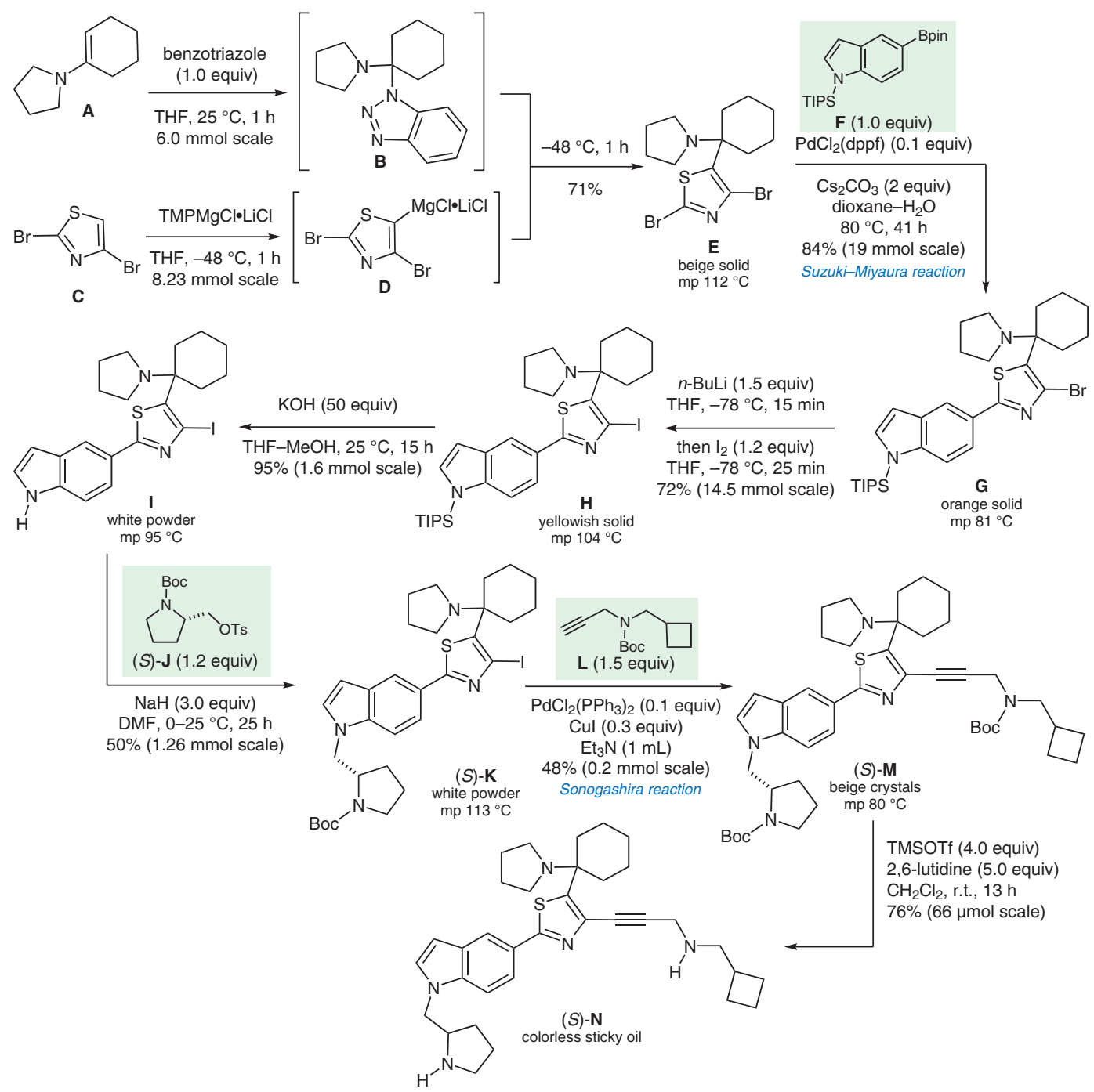

Significance: The parasitic protozoa responsible for trypanosomiasis, Chagas' disease, and leishmaniasis require the reduction of trypanothione disulfide to trypanothione, which the parasites use in several essential processes. Target molecule $\mathbf{N}$ is the strongest competitive inhibitor in vitro of trypanothione reductase from Trypanosoma cruzi reported to date.
Comment: Note the construction of highly hindered amine $\mathbf{E}$ by nucleophilic substitution of benzotriazole from $\mathrm{N}, \mathrm{N}$-acetal B by the organomagnesium reagent $\mathbf{D}$.

Review: For a Review on properties and synthetic utility of $\mathrm{N}$-substituted benzotriazoles, see $\mathrm{A}$. $\mathrm{R}$. Katritzky, X. Lan, J. Z. Yang Chem. Rev. 1998, 98, 409-548.

Synthesis of Natural

Products and

Potential Drugs

Key words

trypanothione reductase inhibitor

benzotriazole

$N, N$-acetal

tertiary amine

Suzuki-Miyaura coupling

Sonogashira coupling 